\title{
ARGONNE PLASMA WAKE-FIELD ACCELERATION EXPERIMENTS
}

DE89 009816

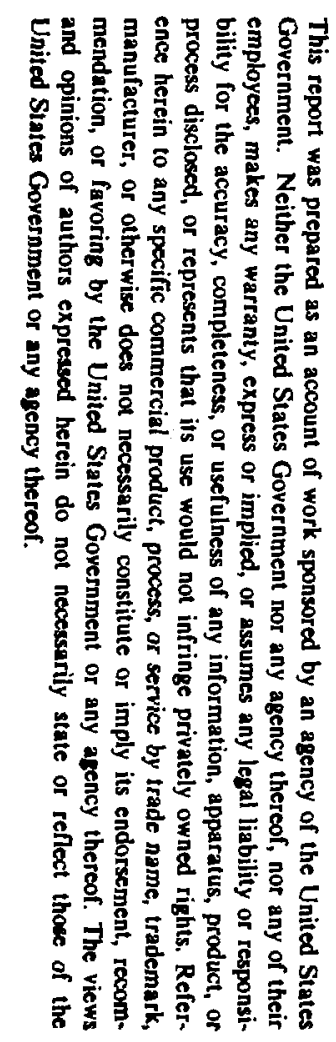

J. B. ROSENZWEIG*, B. COLE, W. GaI, R. KONECNY, J. NOREM, P. SCHOESSOW, AND J. SIMPSON

High Energy Physics Division

Argonne National Laboratory

Argonne, Mlinois 60499

Subrnitted to the Proceedings of the 1989

Lake Arrowhead Workshop on Advanced Accelerator Concepts

*Present address: Fermi National Accelerator Laboratory.

Work supported by the U.S. Department of Energy, Division of

High Energy Physics, Contract W-31-109-ENG-38 


\section{Introduction}

Four years after the initial proposal of the Plasma Wake-field Accelerator ${ }^{[1]}$ (PWFA), it continues to be the object of much investigation, due to the promise of the ultra-high accelerating gradients that can exist in relativistic plasma waves driven in the wake of charged particle beams. These wake-fields are of interest both in the laboratory, for acceleration and focusing of electrons and positrons in future linear colliders, and in nature as a possible cosmic ray acceleration mechanism. ${ }^{|2|}$ The purpose of the present work is to review the recent experimental advances made in PWFA research at Argonne National Laboratory.

Experimental investigations of the PWFA at the Argonne Advanced Accelerator Test Facility ${ }^{[3]}$ (AATF) have produced a multitude of accomplishments in the short time since the last advanced accelerator workshop (Madison, Wisconsin, August 1986). The results of these experiments have directly verified the existence of electron acceleration in plasma wake-fields by the injection of a test bunch of electrons into a beam-driven plasma wave, thus measuring the longitudinal dependence of the accelerating and deflecting wake-fields. These proof-of-principle experiments, ${ }^{\left|{ }^{\mid}\right|}$which will be described below, validated major relevant predictions of linear wake-field theory, ${ }^{[s-7]}$ including the fundamental excitation of the electron plasma waves with their associated longitudinal and transverse electrostatic fields, the electromagnetic self-pinching forces of a high intensity driving beam, and the dependence of these phenomena on beam and plasma characteristics.

After the proof-of-principle experiments were completed, improvements in facility operation allowed a second round of experiments which explored an entirely different regime of the PWFA. High resolution measurements of the wake-fields excited by more intense driving beams revealed large nonlinearities in the wakefields, indicating that the driving beams became significantly self-pinched within the plasma by their transverse wake-fields. ${ }^{(0)}$ Both of the nonlinearity and the selfpinching are exciting observations, as the nonlinear wake-fields can theoretically 
yield large transformer ratios, ${ }^{|0|}$ and the self-pinching wake-fields have been proposed for use in a powerful final focusing lens for a linear $e^{+} e^{-}$collider. ${ }^{[5] \mid 10]}$ The results and analysis of the nonlinear PWFA experiments will also be presented below.

After the discussion of the experimental investigations which have been completed to date, we will turn to the subject of present and future efforts in this field. The present work is concerned with direct measurement of a self-focused electron beam profile in a plasma. Prospects for future experiments, to be performed with a much more intense beam derived from a new laser photo-cathode source at the AATF, will be examined. Theoretical expectations for nonlinear wake-field amplitudes in these experiments will be estimated; they are predicted to be greater than $1 \mathrm{Gev} / \mathrm{m}$.

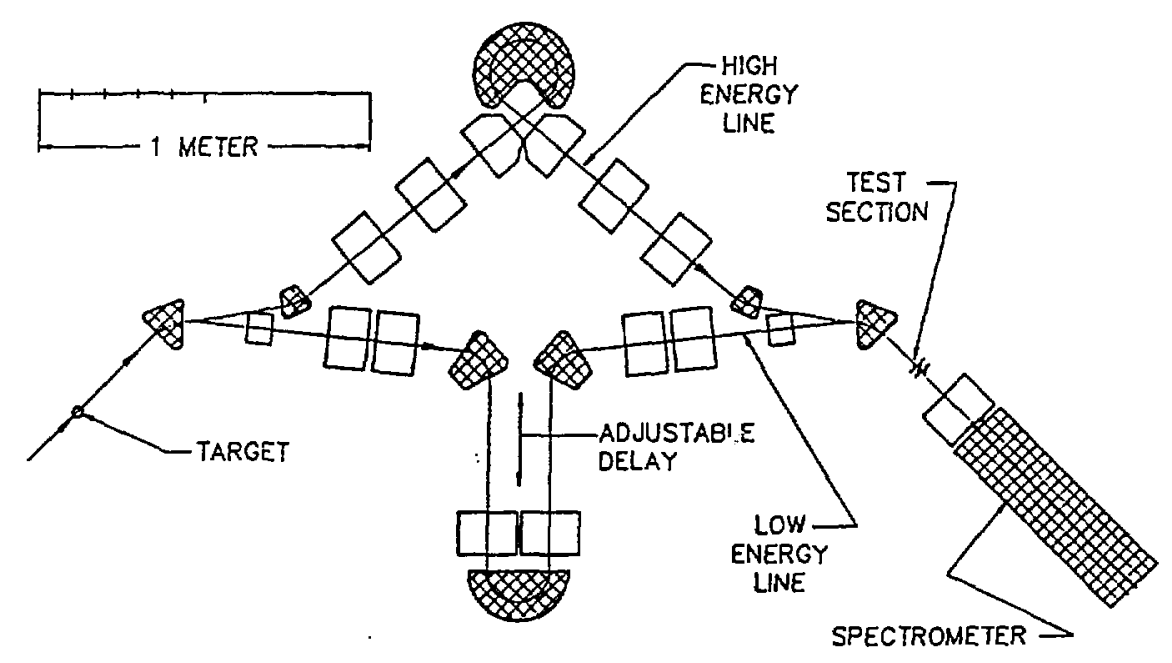

Figure 1: Schematic of Argonne Advanced Accelerator Test Facility layout. 


\section{The Argonne Advanced Accelerator Test Facility (AATF)}

The AATF was constructed, for the purpose of studying the physics of wakefield acceleration, by the Argonne High Energy Physics Division Advanced Accelerator Research Group at the Argonne Chemistry Division $21 \mathrm{MeV}$, intense, short pulse electron linac. Beam pulses delivered from this L-band linac to the entrance of the AATF typically contain $N \simeq 4 \times 10^{10}$ electrons and are of length $\sim 15-25$ psec FWHM. The transverse emittance of these beam pulses is typically $7 \pi$ mm-mrad.

A schematic of the AATF layout is shown in Fig. 1. The high intensity 21 $\mathrm{MeV}$ driving beam pulse traverses the upper beam line. A secondary, low intensity, $15 \mathrm{MeV}$ witness beam, which is created by degrading a portion (typically 20-30 percent) of the driver on a carbon target just prior to the facility entrance, is transported through the lower beam line. The combination of scattering and energy spread in the energy-degraded electon population requires momentum and emittance collimation in the witness beam line; approximately $10^{7}$ electrons per pulse are accepted into this transport line. The witness beam leg contains an adjustable "trombone" section which allows variation of the delay time between driver and witness from -0.2 to greater than 1.0 nanosecond. Both beam lines are designed to be isochronous in order to preserve the bunch time structures. The two beams are recombined at the end of the facility and pass through the wake-field experimental section. The minimum beam beta-function in the experimental section is $\beta^{*} \geq 30 \mathrm{~cm}$, giving a rms transverse beam size $\sigma_{r} \geq 1.4 \mathrm{~mm}$. Both beams are analyzed for energy spectra and transverse deflections by a high resolution $\Delta p / p \simeq 10^{-3}$, broad range spectrometer. The beam profiles at the focal plane of the spectrometer, as well as at the beam position monitors in the AATF, are monitored by CCTV imaging of phosphor plates. Beam currents are measured by use of Faraday cups. A 2-picosecond resolution streak camera allows determination of the beam pulse length at the entrance and exit of the facility, as well as measurement of the beam's longitudinal phase space distribution at an 
up-stream spectrometer magnet. All facility optics systems and data aquisition are under remote computer control. ${ }^{[1]}$

The high intensity and short pulse length of the facility driving beam make it useful for driving plasma waves and their strong wake-fields. However, the crucial, unique experimental tool the AATF possesses is its witness pulse, which allows the probing of the plasma wake-fields at arbitrary positions behind the driver. Although this paper is concerned with description of the PWFA experiments, it should be mentioned that alternative wake-field schemes, employing disk- ${ }^{[3]}$ and dielectric-loaded $^{[12]}$ cylindrical waveguides as a slow-wave structure, have also been investigated experimentally using the unique capabilities of the AATF.

\section{Linear Plasma Wake-field Theory}

The two dimensional linear theory of plasma wake-fields, as formulated by Chen, ${ }^{[s]}$ provides a simple model for calculating expected wake-fields in a PWFA experiment. This model has been used in optimizing the experimental parameters to match the beam characteristics at the AATF. The linear theory developed thus far applies to a cold plasma with density perturbation small compared to the unperturbed electron density, which is a good approximation for the experimental situation encountered in the proof-of-principle experiments.

We begin our discussion of the linear theory by reviewing the notion of a wakefield. Assuming the beam motion is mainly parallel to the $z$-axis, the transverse and longitudinal wake-fields are defined as

$$
\begin{gathered}
\mathrm{W}_{\perp}=q\left(\mathrm{E}_{\perp}+\frac{1}{c} \mathrm{v}_{\mathrm{b}} \times \mathrm{B}\right) \quad \text { and } \\
W_{z}=q E_{z},
\end{gathered}
$$

respectively, and where the $\mathbf{E}$ and $\mathrm{B}$ fields are assumed to be beam generated. If we assume that the wake-fields are a function of the longitudinal coordinate 
and time only through the variable $\xi=z-v_{b} t$, then it is easy to show that $\nabla \times \mathbf{W}=0$ and thus the wake fields can be derived from a potential. This is merely the differential form of the Panofsky-Wenzel theorem. ${ }^{\left[{ }^{[3]}\right.}$ Since $v_{b} \simeq c$ for beams of interest we can write this wake potential as $\Omega=A_{z}-\phi$, where $A_{z}$ is the longitudinal component of the electromagnetic vector potential and $\phi$ is the electrostatic scalar potential. The wake-fields are given in this approximation by $\mathbf{W}=\nabla \Omega$.

From the cold plasma fluid equations with the ions assumed stationary, a linearized equation has been derived ${ }^{|s|}$ which describes small amplitude plasma electron oscillations driven by an extremely relativistic electron beam. The equation can be written as

$$
\frac{\partial^{2} n_{1}}{\partial \xi^{2}}+k_{p}^{2} n_{1}=-k_{p}^{2} n_{b}
$$

where $n_{1}=n-n_{0}$ is the deviation of the plasma electron density from equilibrium density $n_{0}, k_{p} \equiv 2 \pi / \lambda_{p}=\omega_{p} c=\sqrt{4 \pi r_{e} n_{0}}$ is the plasma wave number, and $n_{b}$ is the beam density. The linearization of the equations requires that $n_{1} \ll n_{0}$. All focusing calculations in this regime apply equally well to positron beams, but for the purpose of illustration we discuss electron beans for the remainder of this paper.

If we assume that the beam density distribution can be expressed in separable form as

$$
n_{b}=\rho_{b} f(\tau) g(\xi)
$$

where $\rho_{b}$ is the maximum beam density, $r$ is the radial coordinate, and $\xi$ is the longitudinal coordinate measured from the center of the bunch, the solution of Eq. (3.3) is given by ${ }^{[5]}$

$$
n_{1}=-k_{p} \rho_{b} f(r) \int_{\xi}^{\infty} g\left(\xi^{\prime}\right) \sin \left(k_{p}\left(\xi^{\prime}-\xi\right)\right) d \xi^{\prime} \equiv-\rho_{b} f(r) G\left(k_{p} \xi\right) .
$$

Note that the plasma charge density $-e n_{1}$ has the same transverse dependence 
as the beam density $f(r)$. The longitudinal response, however, is described by $G\left(k_{p} \xi\right)$, a convolution integral over the beam density ahead of the point in question with the causal Green's function solution to Eq. (3.3).

Also notice that for an infinitesimally short driving beam of surface charge density $\Sigma_{b}$ the plasma wave left in the wake of the beam has the form $n_{1}=$ $-k_{p} \Sigma_{b} f(r) \sin \left(k_{p} \xi\right)$. This is the most efficient (impulse) excitation of the plasma wave; if the beam is not short compared to a plasma wave then the amplitude of the excited wave is smaller, as the plasma electrons have time to react to and partially neutralize the beam space charge density. We quantify this effect by defining the wake plasma wave longitudinal excitation efficiency to be

$$
\eta_{l}=\frac{\left\|n_{1}\right\|}{k_{p} \rho_{b} f(r) \int g(\xi) d \xi}
$$

which is the density perturbation amplitude normalized to that obtained for an infinitesimally short driver beam of the same transverse profile. This factor can be calculated by performing the convolution integral in Eq. (3.5). For a beam with a longitudinal Gaussian profile of rms length $\sigma_{z}$, we have

$$
\left.\eta_{l}=\exp \left(-k_{p} \sigma_{z}\right)^{2} / 2\right)
$$

Chen derives the wake potential corresponding to the plasma response by solving the following equation:

$$
\left(\nabla_{\perp}^{2}-k_{p}^{2}\right) \Omega=-4 \pi e n_{1}
$$

This equation yields approximate solutions to the wake-fields which ignore the terms of order $\gamma^{-2}$, i.e. the net force due to the beam vacuum fields. ${ }^{[3]}$ The causal solution to this equation is given by

$$
\Omega=\frac{4 \pi e \rho_{b}}{k_{p}^{2}} F\left(k_{p} r\right) G\left(k_{p} \xi\right)
$$

where $F\left(k_{p} r\right)$ is the radial part part of the solution. The radial part is obtained alternatively from another Green's function integral expression. ${ }^{|s|}$ Note from the 
above discussion that the wake-fields have both electrostatic and electromagnetic components. The homogeneous plasma waves in the wake region are purely electrostatic oscillations. Inside the beam, the electromagnetic portion of the wake-field is due to the self-magnetic field of the beam.

In the limit that the beam is wide compared to the plasma wave length $k_{p} \sigma_{r} \gg$ 1, $F\left(k_{p} r\right) \simeq f(r)$, and the plasma motion and wake-field is mainly longitudinal. In the opposite limit, $k_{p} \sigma_{r} \gg 1$, the plasma motion and wake-field is largely radial. We thus define a radial field efficiency as follows $\eta_{r}=F(0)$ which is near unity for $k_{p} \sigma_{r} \gg 1$ and becomes logarithmically small for $k_{p} \sigma_{r} \ll 1$. The maximum accelerating wake-field driven on axis can now be written in terms of these two geometrical efficiencies,

$$
e E_{z, \max }=4 \pi r_{e} m_{e} c^{2} \Sigma_{b} \eta_{l} \eta_{r}
$$

If the beam is disk-like, with $\sigma_{z} \ll \sigma_{r}$, then a working point in plasma density can be found in which $\eta_{l}$ and $\eta_{r}$ are near unity, and the largest amplitude plasma waves can be driven. On the other hand, if the beam is sausage-like, and $\sigma_{r} \ll$ $1 \ll \sigma_{z}$, the plasma responds to nearly completely neutralize the space charge density of the beam $\left(G\left(k_{p} \xi\right) \simeq g(\xi)\right)$, while the plasma return current flows inside a cylinder of radius $\sim k_{p}^{-1} \gg \sigma_{r}$. Thus the beam's repulsive self-electric force is neutralized while the focusing self-magnetic force is left unbalanced. ${ }^{[13]}$ This focusing, which can be termed the plasma lens effect, is potentially quite powerful and can lead to self-pinching of the beam in the plasma. A self-pinched beam profile can in turn drive larger amplitude plasma waves, as will be emphasized when we discuss the nonlinear PWFA experiments. 


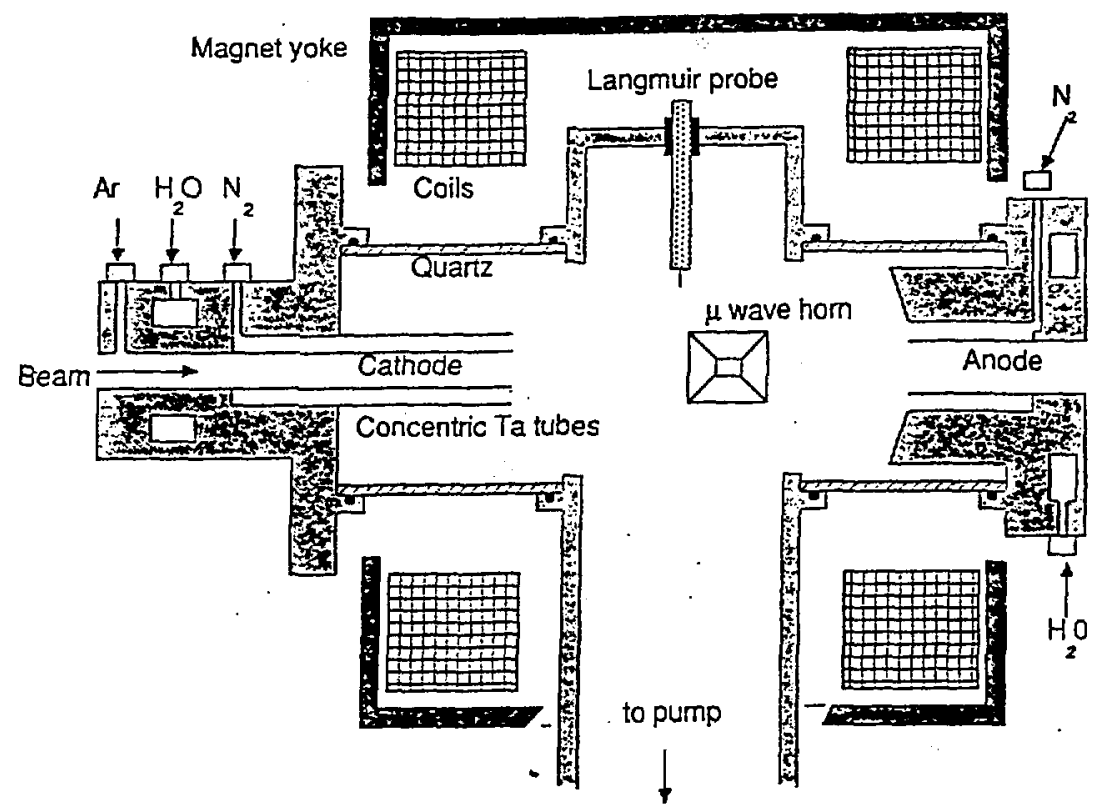

Figure 2: Schematic drawing (not to scale) of hollow cathode arc plasma source used in PWFA experiments

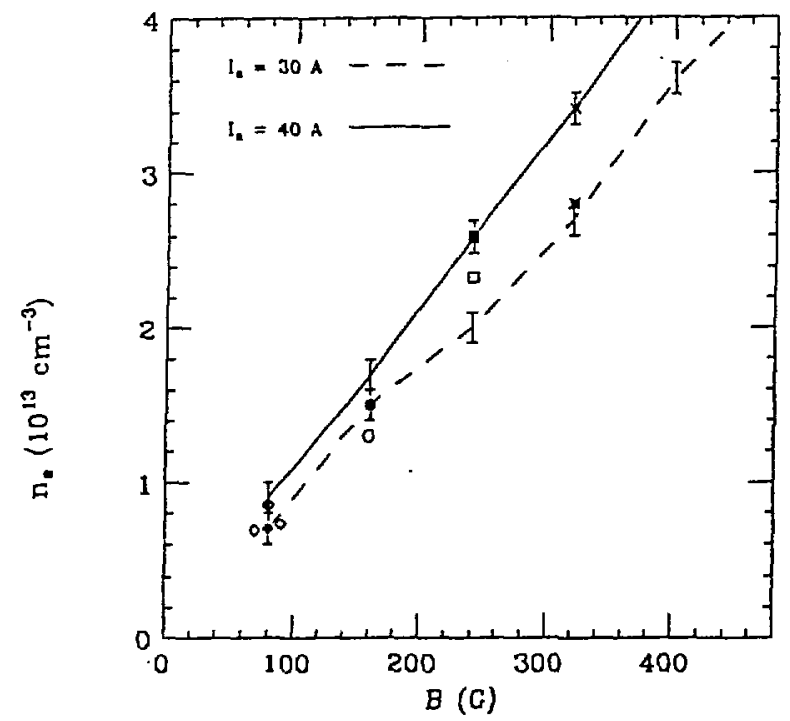

Figure 3: The plasma electron density $n_{e}$ from probe measurements for various running conditions (arc current $I_{a}$ and applied axial magnetic field $B$ ) of the arc source. The darkened symbols show the density as determined from $\omega_{p}$ in PWFA experiments, and open symbols are the corresponding probe measurements. 


\section{Measurement of Linear Flasma Wake-fields}

It is clear from the discussion above that the maximum longitudinal wakefields are generated if $k_{p} \sigma_{z} \ll 1 \ll k_{p} \sigma_{r}$, which means a beam much shorter than it is wide. In the proof-of-principle linear PWFA experiments the beam was approximately round $\left(\sigma_{z} \simeq \sigma_{r} \simeq 2.4 \mathrm{~mm}\right)$ and is thus not quite optimum. The total geometrical excitation efficiency $\eta_{l} \eta_{r}$ for this case has a maximum value of about 0.45 , at a point where $k_{p}$ would indicate use of a plasma density of $n_{e}=8 \times 10^{12} \mathrm{~cm}^{-3}$ for these beam dimensions. We may consider this an optimum point in plasma density to observe large accelerating gradients in the the linear PWFA. Experimentally, there are two complications in this case over a diskshaped beam: the transverse fields are larger relative to the longitudinal fields, and with a witness beam length not small compared to a plasma wavelength, the resolution of the measurement is degraded. In the linear PWFA measurements, the total beam charge per bunch is approximately $2.1 \mathrm{nC}\left(N=1.3 \times 10^{10} e^{-}\right)$.

The DC plasma source used for the PWFA experiment is a hollow cathode arc constructed at the University of Wisconsin. A schematic, not-to-scale drawing of the arc source is shown in Fig. 2. The arc electrical characteristics are typically $V_{a} \simeq 50 \mathrm{~V}$ and $I_{a} \simeq 30-60 \mathrm{~A}$. The interelectrode plasma column length $L_{p}$ is $20-35 \mathrm{~cm}$, and density $n_{e}$ is variable about our optimum working point, froin approximately $0.5-8.0 \times 10^{13} \mathrm{~cm}^{-3}$, with an electron temperature of $2-8 \mathrm{eV}$. An axial magnetic field $50-700 \mathrm{G}$ is applied to confine the plasma column. The feed gas is argon, and the background pressure is limited to approximately 1-2 $\mu \mathrm{m}$ by use of a $750 \mathrm{l} / \mathrm{sec}$ turbo-molecular pump. Plasma diagnostics include microwave interferometry (line integral of plasma density across the column) and electrostatic triple probes. The probe measurements give local measurements of $n_{e}\left(n_{0}\right)$ and $T_{e}$ the density is also determined to excellent accuracy $(\sim 1 \%)$ by the PWFA measurement of the plasma frequency. A comparison of probe and PWFA plasma density measurements is shown in Fig. 3. The probe measurements agree with the more precise and unambiguous PWFA data to within $20 \%$. 
Both the witness and driver beam profiles are monitored on CCTV at the focal plane of the spectrometer. The video images are digitized through use of a frame grabber and stored on disk for off-line analysis. The witness beam data in PWFA experiments is complicated by the large and rapidly fluctuating background of light generated by the driver beam halo which precludes a simple subtraction of a background frame from the data. Instead, an algorithm is employed which extrasts the majority of the witness beam spot from the rest of the image, and calculates the resulting centroids of the image distribution in the dispersive and deflection planes. Background baseline shifts due to changing witness beam transmission optics are then subtracted. Thus, at each point in witness beam delay we determine the centroids of the witness beam distribution in the energy analyzing and deflecting planes. The witness beam wake-field scans in these experiments consist of many such points incremented in delay by fine time steps over a range of many wake-field oscillation lengths.

Witness beam delay scans, displaying beam energy centroid as a function of the time delay between diver and witness beam, ars shown in Figs. 4 (Scan 1) and 5 (Scan 2). The dashed lines indicate the predictions of the linear PWFA theoretical model, plotted for comparison to the data. The theoretical predictions include the experimental effects of the resolution of the witness beam, which we discuss below. These effects make the predictions approximate due to the shotto-shot fluctuation of the beam length and charge measurements.

All theoretical predictions consistent with these uncertainties would form a band about the dashed line shown in each figure. Only the central prediction is shown for clarity. Ahead of the driver, we observe small fluctuations in the energy centroid due to background and linac fluctuations. The centroid then lowers while passing through the region near zero delay, as it feels the decelerating wake on the driver. Behind the driver we observe oscillations characteristic of the plasma wake-fields, harmonic with the plasma frequency. These oscillations persist for many wavelengths, and maintain a nearly constant frequency. In both scans the driver beam charge, measured by using the spectrometer as a Faraday cup, was 
determined to be $2.1 \mathrm{nC}$, with shot-shot fluctuation of approximately $5 \%$. The plasma characteristics were: $\operatorname{scan} 1-L_{p}=28 \mathrm{~cm}$ and $n_{e}=8.6 \times 10^{12} \mathrm{~cm}^{-3}$, and scan $2-L_{p}=33 \mathrm{~cm}$ and $n_{e}=2.3 \times 10^{13} \mathrm{~cm}^{-3}$. The densities quoted above were deduced from FFT calculation of the oscillation frequency.

The maximum accelerating wake-field amplitude $W_{m}$ can be calculated from the maximum energy centroid excursions $\delta E_{m}$. We must first average over the longitudinal wake-field felt by different parts of the witness beam spatial distribution, and divide by the resulting resolution function. This function can be divided into two factors, a radial resolution function $R_{r} \simeq \sigma_{r, d}^{2} /\left(\sigma_{r, w}^{2}+\sigma_{r, d}^{2}\right)$, where $\sigma_{r, d(w)}$ is the radial beam size of the driv resolution function $R_{l}=\exp -\left[\left(k_{p} \sigma_{z}\right)^{2} / 2\right]=\eta_{l}$. For both scans the radial resolution $R_{r}$ is about 0.5 , but with the higher density in scan 2 one obtains less longitudinal resolution due to the shortening of the plasma wavelength. For the beam and plasma characteristics of Scan $1, R_{l}=0.43$, and for Scan $2, R_{l}=0.11$. The maximum accelerating wake-field is thus given by the expression

$$
W_{m}=\frac{\delta E_{m}}{R_{r} R_{l} L_{p}}
$$

which gives $W_{m} \simeq 0.94 \frac{ \pm 0.27}{-0.19} \mathrm{MeV} / \mathrm{m}$ for Scan 1 and $1.60 \frac{+0.63}{-0.30} \mathrm{MeV} / \mathrm{m}$ for Scan 2. The uncertainty in the determination is due mainly to the $20 \%$ uncertainty in the beam length (as determined by streak camera measurements) from shotto-shot, which strongly affects the calculated wake-field amplitudes through the quickly varying longitudinal resolution function. The agreement with the simple theoretical model is quite good, as indicated in Figs. 4 and 5.

Scanning further back in delay shows a decay in amplitude of the measured energy centroid shifts. Since the plasma is not collisional ( $\omega_{p} \gg \nu_{e-i}$, the collision frequency), we attribute this decay to the longitudinal density gradient along the plasma column. The phase delay between the two beams is related to the time delay by $\Delta \phi=\omega_{p}(z) \Delta t$, so that a variation in the plasma density changes the phase delay more dramatically at larger time delays. These larger changes in 
the phase delay cause the total wake-fields integrated by the witness beam to be diminished. Assuming the plasma density gradient along the column is small and constant, the total density variation $\delta n_{e} / n_{e}$ in the plasma can be related to the observed damping of the measured wake-field amplitude $\delta W_{m} / W_{m}$ by

$$
\frac{\delta W_{m}}{W_{m}} \simeq\left|\operatorname{sinc}\left(\frac{\omega_{p} \Delta t}{4} \frac{\delta n_{e}}{n_{e}}\right)\right|
$$

For a scan shown in a following section (Scan 4), in which we observed a degradation in amplitude $\delta W_{m} / W_{m} \simeq 0.6$ after eighteen plasma wavelengths of delay we deduce a density variation of about $6 \%$. This also is consistent with electrostatic probe measurements.

The existence of strong transverse plasma wake-fields is of great interest due to their possible application as a final focusing scineme for linear coliiders. (This subject is explored in great detail in the literature. ${ }^{|s|[10][15][16.17]}$ ) Evidence for such forces were observed in transverse deflections in the witness beam non-bend plane position centroid when the witness beam and driver were not collinear. One such scan (Scan 3) is shown in Fig. 6, which plots the position centroid versus delay time. For this scan the driver beam charge is $2.2 \mathrm{nC}$, the plasma length is $33 \mathrm{~cm}$ and the plasma density is $n_{e}=3.4 \times 10^{-13} \mathrm{~cm}^{-3}$. The deflection is quite strong at zero delay, where the driver magnetic fields provide a strong pinching force. The plasma excitation efficiency is very low for this case due to the high plasma density, as the plasma lens regime has been entered, so the focusing wake-fields are expected to be non-negligible only inside the driver, as is observed. The $5 \mathrm{~mm}$ maximum position change on the focal plane corresponds to approximately $2.5 \mathrm{mrad}$ of deflection, or $37.5 \mathrm{keV}$ of transverse kick, which is of the same order of magnitude as the acceleration amplitudes observed in the linear on-axis scans.

A typical driver beam energy loss spectrum is shown in Fig. 7, with a comparison to the case of no plasma present. Qualitatively, we observe the most total driver energy loss at lowest plasma density, which is expected, as the total energy 
loss should scale as $\eta_{r} \eta_{l}^{2}$, and $\eta_{l} \sim \exp \left(-n_{0}\right)$. However, we are unable to quantify the total energy loss well with the present spectrometer geometry, because the self-pinching of the decelerating driver beam electrons deflects a portion of the beam distribution off the focal plane detectors. This is further evidence, of course, for the strong focusing wake-fields inside the driver beam. 


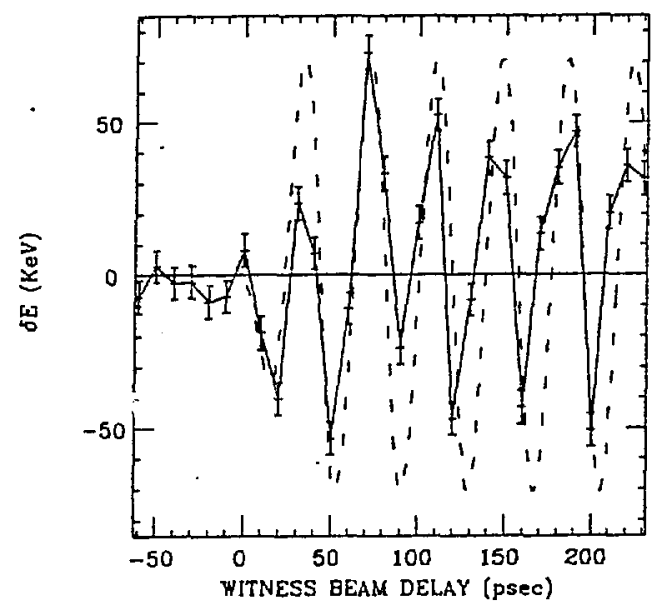

Figure 4: Scan 1 - Witness beam energy centroid change $\delta E$ versus time delay behind driver. Total driver beam charge $Q=2.1 \mathrm{nC}$, plasma parameters $L_{p}=28 \mathrm{~cm}$ and $n_{e}=8.6 \times 10^{12} \mathrm{~cm}^{-3}$. Theoretical predictions are given by the dashed line.

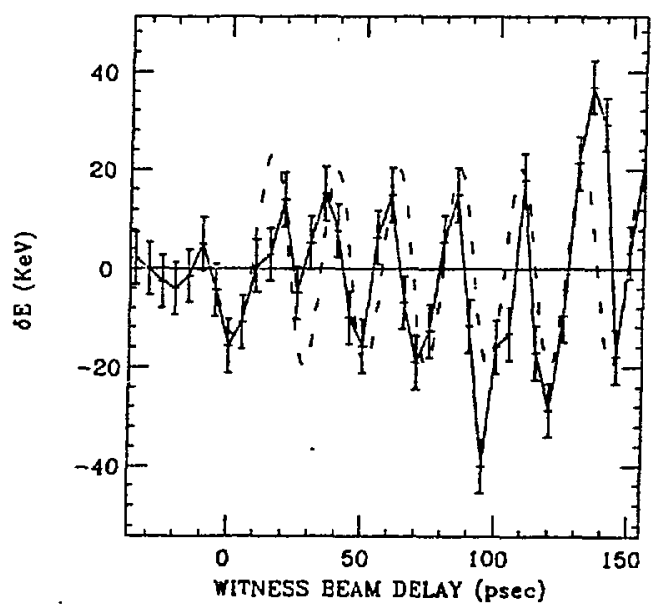

Figure 5: Scan 2 - Witness beam energy centroid change $\delta E$ versus time delay Total driver beam charge $Q=2.1 \mathrm{nC}$, plasma parameters $L_{p}=33 \mathrm{~cm}$ and $n_{e}=2.3 \times 10^{13} \mathrm{~cm}^{-3}$. Theoretical predictions are given by the dashed line. 


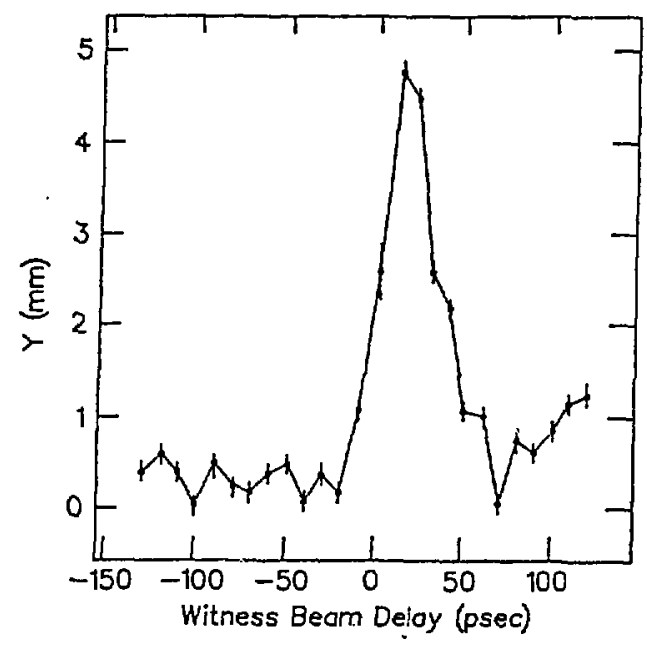

Figure 6: Scan 3 - Witness beam centroid position $\Delta y$ in the non-bend plane of the spectrometer versus delay, for off-axis injection of witness beam. The large deflection near zero delay is expected from the linear theory, due to the self-focusing magrietic wake-fields at the driver. For this $\operatorname{scan} Q=2.2 \mathrm{nC}$, $L_{p}=33 \mathrm{~cm}$ and $n_{c}=3.4 \times 10^{-13} \mathrm{~cm}^{-3}$.

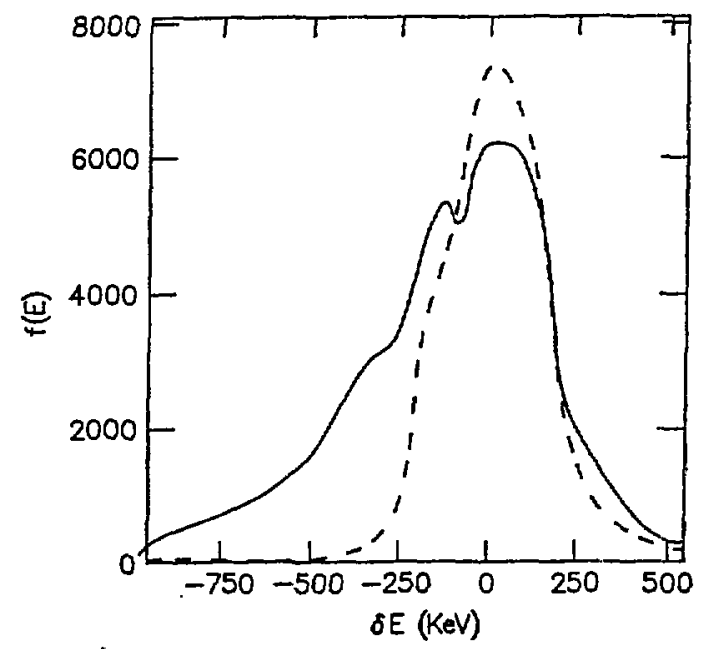

Figure 7: Driver beam energy spectrum for $Q=2.5 \mathrm{nC}, L_{p}=28 \mathrm{~cm}$, and $n_{e}=2.9 \times 10^{-13} \mathrm{~cm}^{-3}$ plotted with solid line, along with case of no plasma present in dashed line. 


\section{Review of Nonlinear Plasma Wave Theory}

Much of the theoretical effort to date has concentrated on the linear regime of the PWFA, in which assumption of small amplitude electron density perturbations $\left\|n_{1} / n_{0}\right\| \ll 1$ allows linearization of the fluid equations. The predominance of linear theory summarized above is due to its usefulness - it allows calculation of the general three-dimensional variation of the transverse and longitudinal wake-fields if the driving electron beam current distribution is a known quantity, unchanging in time. This linear treatment is of course inadequate to deal with self-pinching of the driver beam, ${ }^{[14]}$ the effects of which have been previously simulated for early proposed experimental scenarios. ${ }^{[1 \mid \boldsymbol{}}$ In addition, previous theoretical analyses of the nonlinear regime of the PWFA have treated only the one-dimensional case, ${ }^{[0 \mid[10-21\}}$ due to the mathematical intractability of the three-dimensional problem.

For extremely large amplitude plasma waves driven by electron beams the theoretical models developed thus far predict many interesting physical phenomena. The most intriguing is the aforementioned possibility of obtaining high transformer ratios by driving waves with a long electron beam $\left(l_{b} \gg \lambda_{p}\right)$ of density $n_{b}=n_{0} / 2 ;$ the peak value of $n_{1} / n_{0}$ in the wake of such a beam can be much greater than unity. In addition, the problems of background electron trapping and thermal effects, ${ }^{[10][20]}$ ion motion, and continuous beam loading ${ }^{[22]}$ have all been explored using the one-dimensional theory.

For moderately nonlinear one-dimensional waves (the plasma electrons become only slightly relativistic ${ }^{(19)}$ ), steepened wave profiles develop which can be decomposed into Fourier harmonics of the fundamental (plasma) frequency. The harmonic decomposition of the perturbed plasma electron density can be written as $^{[23]}$

$$
\frac{n_{e}-n_{0}}{n_{0}}=\sum_{m=1}^{\infty}\left(\frac{n_{1}}{n_{0}}\right)^{m} \frac{m^{m}}{2^{m-1} m !},
$$

where $n_{1} / n_{0}$ is henceforth taken to be the amplitude of the fundamental compo- 
nent of the electron plasma wave. Nonlinear beat-wave excited plasma waves have been indirectly observed experimentally ${ }^{[23]}$ by laser scattering off these harmonics in the electron density perturbation and predicted in computer simulations of plasma wake-fields. ${ }^{|18|}$

A one-dimensional treatment of the nonlinea- TWFA is expected to be valid only if the driving beam is transversely uniform in density and wide compared to a plasma wavelength. We have already seen from the linear experiments that this is not the case for beams at the AATF. As there exist no two- or three-dimensional nonlinear treatments of the PWFA, and since we predict quite dramatic changes in the transverse profile of the driving beam, we must therefore employ a hybrid of linear and nonlinear plasma wave theories, as well as plasma focusing theory to understand the experimental results we present below. Using theoretical arguments originally derived for the study of beam dynamics ${ }^{[10]}$ in the plasma lens, we can estimate the expected reduction in beum size for the beam and plasma conditions present, in order to address the issue of driver beam pinching analytically.

This predicted pinched beam profile can then be used in the linear approximation to calculate the amplitude of the fundamental frequency excitation of the plasma wake-fields from Eq. (3.5). Using the harmonic decomposition of the one-dimensional density wave in Eq. (5.1) as a basis for a perturbation treatment, we are able to estimate the expected amplitude of the higher harmonics in the wake-fields. Use of the nonrelativistic results is justified even for fairly large $n_{1} / n_{0}$ in our case, as plasma electron velocities for radially dominated waves are not nearly as large as for wide beams of equivalent $n_{1} / n_{0}$. Despite the lack of rigor in the perturbation treatment, $t$ aralysis will be shown to give good qualitative agreement with the experimental results. 


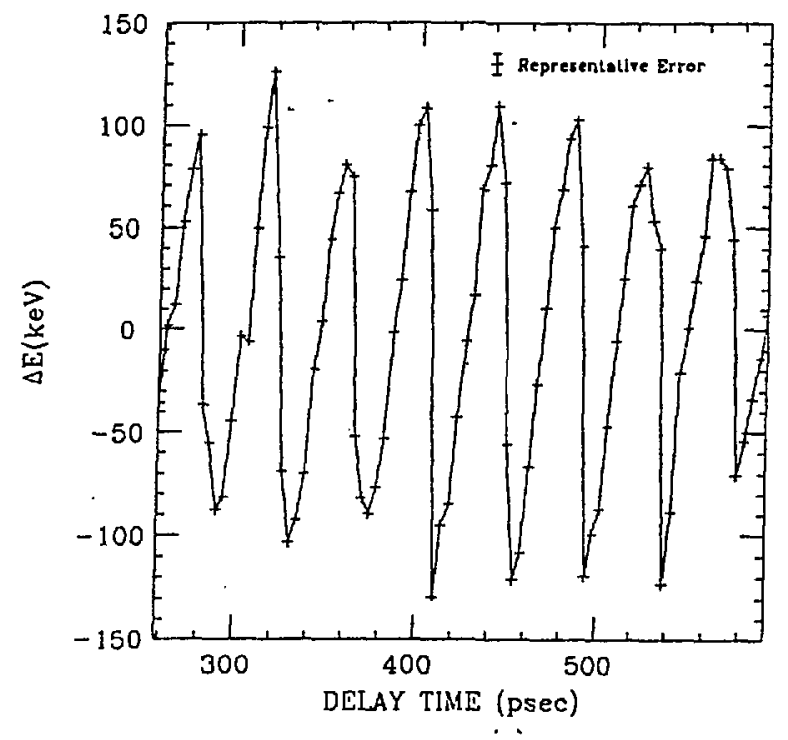

Figure 8(a): Scan 4 - Longitudinal wake-field scan, witness beam energy centroid $\Delta E$ motion versus time delay, with plasma density of $n_{0}=7.3 \times 10^{12}$ $\mathrm{cm}^{-3}$.

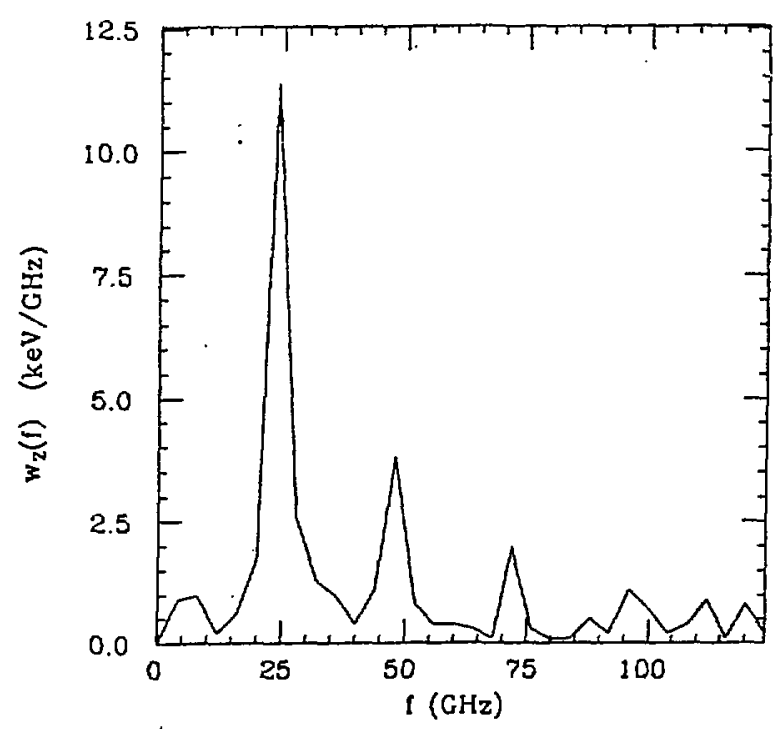

Figure 8(b): FFT amplitude function $w_{z}(f)$ for longitudinal wake-fields. 


\section{Experimental Measurement of Nonlinear Plasma Wake-fields}

In the second round of PWFA experiments performed at the AATF, several changes in the experimental scenario allowed observation of plasma wake-fields with the clear signature of nonlinearity: wave steepening, with its accompanying harmonic generation. In particular, the raising of the driving beam current by a factor of two, coupled with the tighter focusing of the driver and higher resolution measurements (scans finer in time step) of both longitudinal and transverse plasma wake-fields yield dramatic demonstrations of the nonlinear effects.

The $21 \mathrm{MeV}$ driving electron. beam pulse used in the nonlinear experiments has the following characteristics: number of electrons per pulse $N=2.5 \times 10^{10}$ $(Q=4 \mathrm{nC})$, rms pulse length $\sigma_{z}=2.1 \mathrm{~mm}$, and initial rms radius $\sigma_{r}=1.4 \mathrm{~mm}$. The plasma source length was set at $L_{p}=33 \mathrm{~cm}$, and variable plasma densities were explored, as before.

To illustrate most of the observed relevant nonlinear PWFA phenomena we show two high resolution wake-field scans. Scan 4, shown in Figs. $8-10$, is taken with a relatively low plasma density $n_{0}=7.3 \times 10^{12} \mathrm{~cm}^{-3}$. The witness and driver beams are horizontally misaligned in this scan to allow observation of the longitudinal dependence of the transverse wake-fields. Several qualitative remarks can be made upon inspection of Figs. 8(a) and 9. The first is that both the longitudinal and transverse wake-fields are stable, oscillatory functions of the distance behind the driving beam $\zeta=c t-z$. This is surprising, as narrow nonlinear plasma wave may be subject to differences in frequency as a function of transverse position. Secondly, the longitudinal wake-fields $W_{z}$ have taken on a more saw-tooth appearance, as would be expected from the one-dimensional nonlinear theory. The transverse wake-fields show a form consistent with the differential form of the Panofsky-Wenzel theorem, which implies that $W_{x}=\partial_{z} \int_{0}^{r} d r W_{r}$ for a cylindrically symmetric driver. It is apparent from inspection that the measured longitudinal wake-fields are to a good approximation proportional to the longitudinal derivative of the measured transverse wake-fields. In addition to these 
qualitative remarks on the wave-forms, we have found it of use to employ the the Fourier spectrum of the longitudinal wake-fields in attempting to quantify the physical basis for the nonlinearity of these waves using our perturbation treatment. The FFT of the longitudinal wake-field shown in Fig. 8(a) is displayed in Fig. 8(b); note that the ratio of first harmonic to the fundamental amplitude in the wake-fields is about 0.3 .

Using Eq. (5.1) for the amplitude of the $m$-th wave harmonic, the longitudinal electric field on axis associated with this charge density wave is, ignoring phase factors,

$$
E_{z}=\sum_{m=1}^{\infty} E_{m} e^{i m k_{p} s}=4 \pi e n_{0} \sum_{m=1}^{\infty} \frac{n_{m}}{n_{0}} \cdot \frac{\eta_{r m}\left(m k_{p} \sigma_{r}\right)}{m k_{p}} e^{i m k_{p} s}
$$

where $\eta_{r m}\left(m k_{p} \sigma_{r}\right)$ is the radial efficiency factor defined a.bove, generalized for all the wave harmonics. This factor is a monotonically increasing function of its argument, and thus is an increasing function of harmonic number $m$. The ratio of first harmonic $E_{2}$ to fundamental $E_{1}$ amplitude is simply $1 / 2\left(n_{1} / n_{0}\right)\left(\eta_{r 2} / \eta_{r 1}\right)$. This implies, for example, that the density perturbation at the fundamental frequency in $\operatorname{Scan} 4$ is $n_{1} / n_{0} \simeq 0.33$.

This wave amplitude is not consistent with the predictions of linear theory if one ignores possible pinching of the driver beam. This is easily seen, as in the linear theory the wake wave amplitude for a bi-Gaussian driver is given by ${ }^{[\omega],|7|}$ $n_{1} / n_{0}=2 N r_{e} \exp \left[-\left(k_{p} \sigma_{z}\right)^{2} / 2\right] / k_{p} \sigma_{r}^{2}$. For the present case, with $\sigma_{r}$ taken as its initial value, this yields $n_{1} / n_{0}=0.08$, which is much smaller than the estimate from harmonic content. Thus we are led to suggest that significant self-pinching of the driver mist have occurred.

In order to further support this hypothesis, we compile a list of observations. The first, of course, is the previously discussed measurement of large witness beam deflections at zero delay, which indicate the existence of large focusing fields. Also recall our discussion of the expansion of the driver beam image 
' in the spectrometer nonbend plane with plasma present, which implies strong overfocusing of the beam in front of the focal plane. In addition, we note that the driving beam is aperture limited by the plasma - node with the plasma source off, and $20 \%$ of the beam current is not transmitted to the spectrometer. When the plasma is turned on, the full current is transmitted through the previously limiting aperture. Finally, we can examine a different range of Scan 4, shown in Fig. 10, one in which the beam ch.arge is diminished a factor of 0.72 from 4.0 to $2.9 \mathrm{nC}$, and we observe a disproportionate reduction in the wake-field amplitude, to a factor of approximately 0.35 . This indicates that the pinch effect has been taken below a threshold for significant reduction in beam size, with its resultant enhancement of the wake-field amplitude. Note also that the longitudinal wakefields are much more sinusoidal at the lower amplitudes, and that the measured and predicted amplitudes (assuming no driving beam pinching) of $n_{1} / n_{0}$ in this case are 0.09 and 0.06 , respectively, in much closer agreement than with the full beam charge present.

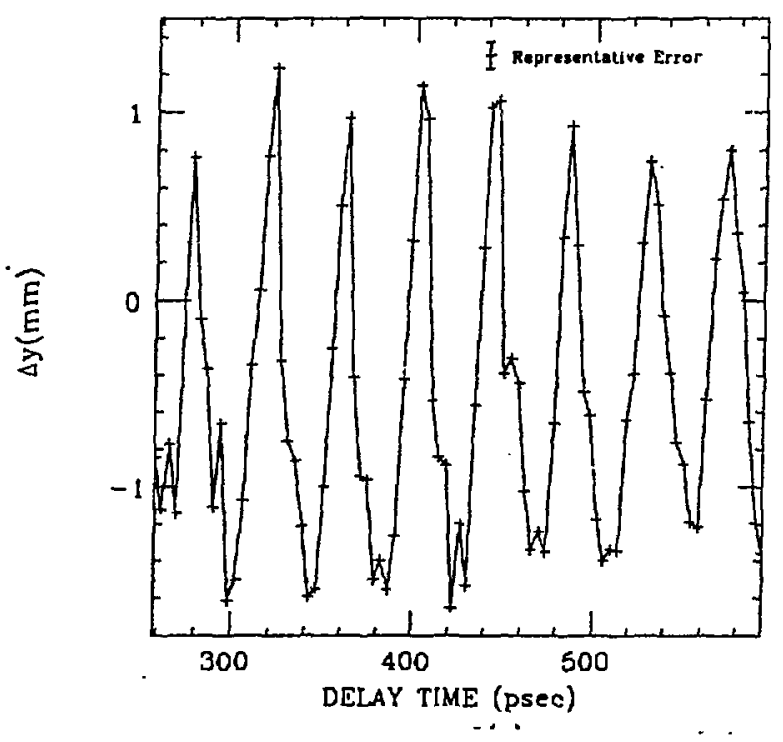

Figure 9: Transverse wake-field scan - witness beam deflection plane $\Delta y$ centroid versus time delay for Scan 4. 
To study beam self-pinching in greater detail, we move on to the analysis of a Scan 5, shown in Fig. 11, in which the plasma is nearly four times as dense as in Scan 4. The norlinearity of these waves is even more pronounced than in the previous scan. The plasma wavelength in this case is twice as short as before $(0.65$ $\mathrm{mm}$ ), and is now shorter than the full beam length $4 \sigma_{z}=0.84 \mathrm{~mm}$. This case is, as in the linear scan shown in Fig. 6 , in the regime of the plasma lens, where the self-focusing forces on the beam can be well approximated by assuming total space charge neutralization of the beam and evaluating the magnetic self-forces of the electron beam distribution. Although this produces non-ideal focusing forces, an approximate envelope equation for the amplitude or $\beta$-function $\left(\beta=\sigma_{r}^{2} / \epsilon\right)$ can be derived that gives some insight to the beam dynamics. ${ }^{[10]}$ We are interested in two quantities from this treatment: the distance inside the plasma required to achieve a pinch (one-half of a betatron wavelength) $s$ and an equilibrium pinched beam spot size $\sigma_{\text {eq }}^{2}$. In terms of the initial value of the $\beta$-function $\beta_{0}=0.3 \mathrm{~m}$ and the invariant $\zeta_{0}=N r_{e} / \sqrt{4 \pi} \epsilon_{n} \sigma_{z}=35 \mathrm{~m}^{-1}$, where $\epsilon_{n}$ is the normalized emittance, we have $\sigma_{e q}^{2}=\epsilon / s_{0}=(0.44 \mathrm{~mm})^{2}$ and $s \simeq \pi / 2 \sqrt{\beta_{0} / \mathrm{s}_{0}}=14 \mathrm{~cm}$. Thus we expect the beam to pinch to approximately one-third its original radius well within the plasma column. This pinchirg is not uniform along the length of the beam, as in our model it depends on the enclosed current at a given point in $\xi$, and we can reflect by writing the invariant as a function of $\xi$, that is $\zeta(\xi)=\varsigma_{0} \exp \left(-\xi^{2} / 2 \sigma_{z}^{2}\right)$. We then have $\sigma_{e q}(\xi)^{-2}=\left(\varsigma_{0} \xi\right) \exp \left(-\xi / 2 \sigma_{2}^{2}\right)$, and the equilibrium density profile on axis is proportional to $\exp \left(-\xi / \sigma_{z}^{2}\right)$, i.e. the bunch is effectively shortened on axis by a factor of $\sqrt{2}$.

The FFT of the scan in Fig. 11 gives a relative first harmonic amplitude value of $E_{2} / E_{1} \simeq 0.48$. The predicted value, using $\sigma_{r}=\sigma_{\ell q}=0.44 \mathrm{~mm}$ and $\sigma_{z}=2.1 / \sqrt{2} \mathrm{~mm}$ in the linear response formula, is $E_{2} / E_{1}=0.38$, in excellent agreement when compared to the prediction assuming no self-pinching of $E_{2} / E_{1}=0.04$. In the first scan, the equilibrium pinched beam radius necessary for the theoretical estimate to agree with the measured harmonic content $\sigma_{e q}=0.77 \mathrm{~mm}$ is not as small as our calculated value for the second scan. This is 
due to the fact that the beam length is shorter than the plasma wavelength, and the beam does not encounter maximum strength transverse wake-fields, as the plasma does not react quickly enough to completely neutralize the beam charge. The driving beam thus takes longer to pinch and does not achieve such small spot sizes.

If we apply the same deconvolution analysis as before, using Eq. (4.1) to give the maximum accelerating wake-field amplitude, we obtain for Scan $4 W_{m}=1.48$ $\mathrm{MeV} / \mathrm{m}$, and for Scan $5 W_{m}=5.3 \mathrm{MeV} / \mathrm{m}$. In the presence of large nonlinearities, transverse forces and the transverse motion induced by these forces, we expect this analysis to be much less reliable, however. Simulations performed at $\mathrm{UCLA}^{[24]}$ indicate that the maximum average accelerating gradient for the conditions in Scan 5 is $7 \mathrm{MeV} / \mathrm{m}$, in fairly good agreement with the experimental result. The prediction for Scan 4 is more problematic, however, as the simulation predicts a maximum average accelerating gradient approximately one order of magnitude greater than we have calculated from the data. This discrepancy is probably due to a combination of effects which are difficult to quantify. This difficulty is in part due to a lack of complete diagnosis of the witness beam distribution in this scan, as good measurement of maximized intensity driver beam is not compatible with the sensitive witness beam diagnostics. Thus the degree of misalignment, and the transverse witness beam profile are uncertain in this scan. The effects of the transverse motion in these wake-fields provide further uncertainty. Another, related source of diminished wake-field amplitude measurement is the probable loss of the tails of the witness beam distribution either in the background level of the focal plane, or completely off the focal plane. All these uncertainties mainly impact on the normalization of the amplitude of the wake-field measurement; they do not greatly affect our conclusions concerning the form (harmonic content) or stability of the longitudinal and transverse nonlinear wake-fields in this scan. The observed form of the wake-fields is reproduced well in the simulations. The stability of the plasma oscillations in the simulations appears to be limited by numerical, rather than physical. 
We have dealt successfully with the data here in a rather phenomenological manner. A more rigorous theoretical understanding of the physics issues would be desirable. The main questions raised by this experiment have to do with the nonlinear plasma response. In these experiments, the beam is much narrower than a plasma wavelength, and thus the plasma electron response and associated wake-fields cannot be well explained by a one-dimensional theory. Conversely, one-dimensional theory predicts improve 1 transformer ratios in very nonlinear waves driven by long, wide beams of density $n_{0} / 2$; it is not possible to confirm this prediction by extrapolating our results. On the other hand, the status of the theory and simulation of beam focusing in plasmas is more well understood than the three-dimensional nonlinear plasma motion. These experimental results are consistent with the theoretical picture of the self-pinch dynamics, and can be viewed as a successful test of the concept of the thick plasma lens, as the peak beam density is increased by a factor of ten inside the plasma. ${ }^{[10]}$

In one-dimensional nonlinear theory, one would expect plasma electron relativistic effects to cause an increase in the plasma period in waves as steepened as we have presented here. One would expect less severe relativistic effects in our cases, however, as the the waves are predominately radial, which causes less energy to be transferred to the plasma electron motion and a concommitant degradation of the longitudinal wake-field amplitude. In fact, we did not observe significant dependence of the plasma wave frequency in this and subsequent experiments. A related aspect of these experiments is that the longitudinal and transverse wake-fields are quite well behaved under these conditions of moderate nonlinearity - the wake-1. . . in Scan 4 were observed out to 18 wavelengths behind the driver with little degradation in form or amplitude. A wave frequency dependence on transverse position, which would be due to relativistic plasma effects, would tend to cause incoherence which could destroy the wake plasma waves. The stability of the observed wake-fields also argues for the absence of relativistic effects in these narrow, nonlinear plasma waves. 


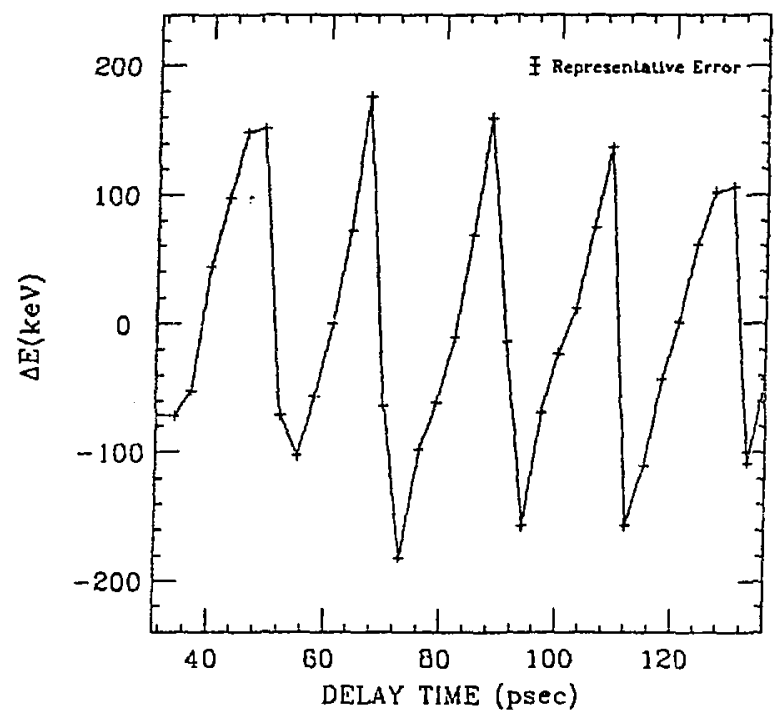

Figure 10: Longitudinal wake-field for Scan 4, with a different delay range. Below $250 \mathrm{psec}$ in delay, the driving beam charge is $2.9 \mathrm{nC}$. Between 250 and 280 the charge is raised to $4 \mathrm{nC}$, where it remains for the rest of the scan. The disproportionate rise in the wake-field amplitude is attributable to stronger selfpinching of the driver beam.

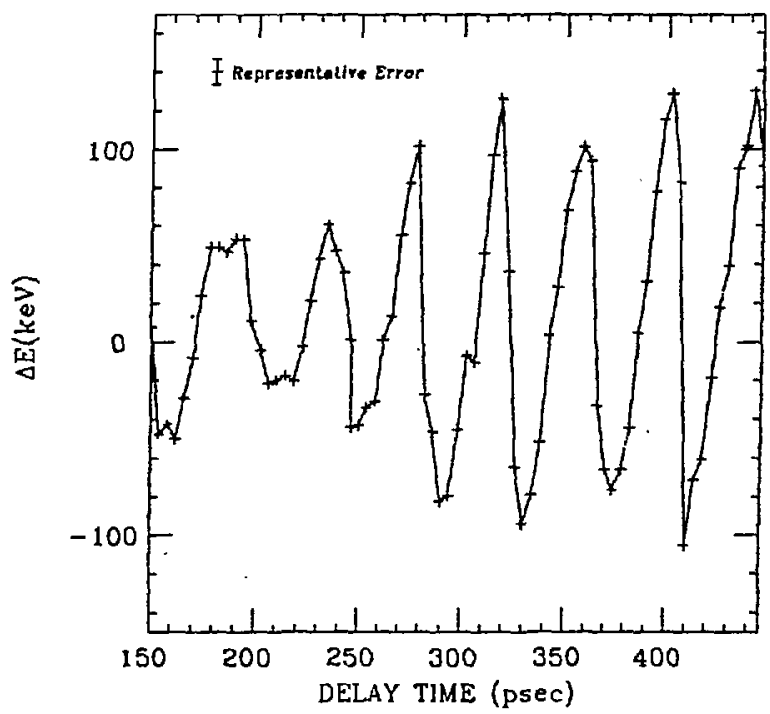

Figure 11: Scan 5 - Longitudinal wake-field scan, with plasma density of $n_{0}=2.8 \times 10^{13} \mathrm{~cm}^{-3}$. 


\section{Present and Future Experiments}

While all of the evidence presented above makes an excellent circumstantial case for the self-pinching of the driving beam in plasma, it is of great interest to verify this pinching directly. To this end, we have designed and implemented a streak camera-based diagnostic that allows measurement of the transverse profile of the beam, as a function of longitudinal position in the beam. Physically, this scheme entails placing an opaque thin walled (to minimize multiple scaitering effects) Cerenkov cell in direct contact with the anode end of the plasma coiumn. The design of this cell is heavily constrained by the difficulty of survival of this relatively fragile device in such a hostile environment as inside of an arc electrode. The photon profile obtained from the cell is focused onto the horizontal slit of the streak camera, giving a sweep image that reflects the transverse profile of the beam in the horizontal direction versus time in vertical (streak) direction. These measurements are on-going as of this writing.

For the future, upgrades of the AATF are planned which should greatly enhance the intensity and brightness of the driving beams available for future wake-field studies. These improvements include a RF laser photocathode source which should provide $100 \mathrm{nC}$ of electrons per pulse in 6 psec FWHM. If this source is used at the Chemistry linac, then the final driving beam energy can be raised to $30 \mathrm{MeV}$. Alternatively, a new $\mathrm{RF}$ acceleration system may be built as part of a general upgrade, which would allow a driving beam energy of 150 $\mathrm{MeV}$. It is of interest to extrapolate the results obtained in the nonlinear PWFA experiments to predict what sort of accelerating gradient can be expected in an experiment at the upgraded facility.

From plasma lens theory, we know that for a beam to efficiently self-pinch in th? plasma its length must be at least on the order of a plasma wavelength. Experimentally, we deduced very powerful self-pinching forces and observed large accelerating gradients in the case where $k_{p} \sigma_{z} \simeq 2$, which is on the border of this regime. The longitudinal efficiency for plasma wave excitation a Gaussian 
longitudinal profile with $k_{p} \sigma_{z} \simeq 2$ is $\eta_{l} \simeq 0.125$. For the purpose of simplifying the remainder of the discussion we will take $k_{p} \sigma_{z}=2$ and $\eta_{l}=1 / 8$.

The equilibrium beam size for a self-pinched beam is given by $\sigma_{r, e q}^{2}==$ $2 \pi^{1 / 2} \epsilon_{n}^{2} \sigma_{z} / \gamma N r_{e}$. For self-pinching to be significant inside the plasma the interaction length $L_{p}$ must be greater than one-half of a betatron wavelength, or $L_{p}>\pi / 2 \sqrt{\beta_{0} / 5}$, where $\beta_{0}$ is the initial beam $\beta$-function. If this condition holds, then the maximum accelerating field behind the beam is approximately given by

$$
W_{m}=\frac{N r_{e} m_{e} c^{2} \eta_{r}\left(k_{p} \sigma_{r, e q}\right)}{4 \sigma_{r, e q}^{2}}=\frac{N^{2} r_{e}^{2} \gamma m_{e} c^{2} \eta_{r}\left(k_{p} \sigma_{r, e q}\right)}{8 \pi^{1 / 2} \epsilon_{n}^{2}}
$$

where $\eta_{\tau}\left(k_{p} \sigma_{r, e q}\right)$ is the radial field efficiency of the in the case of the self pinched beam. Note that the wake-field amplitude appears to have an $N^{2}$ dependence, which is quite dramatic. For cases of interest, however, we often have $\sigma_{r, e q} \ll \sigma_{z}$ and thus $k_{p} \sigma_{r, e q} \ll 1$, a regime where $\eta_{r}\left(k_{p} \sigma_{r, e q}\right) \ll 1$. In this limit Eq. (7.1) becomes

$$
W_{m}=\frac{r_{e} m_{e} c^{2}}{2 \sigma_{z}^{2}}\left[N \ln N-N \ln \left(4 \frac{\sqrt{\pi} \epsilon_{n}^{2}}{\gamma r_{e} \sigma_{z}}\right)\right] .
$$

Now the leading functional dependence of the wake-field amplitude on particle beam population at large $N$ is $N \ln N$, which looks suspiciously like an entropy! As an example, we take the recent experiments, with $\gamma=42, \epsilon_{n}=3 \times 10^{-4}$ m-rad, $N=2.5 \times 10^{10}$, and $\sigma_{z}=2.1 \mathrm{~mm}$, and calculate $W_{m}=6 \mathrm{MeV} / \mathrm{m}$. The experimentally determined value is $W_{m}=5.3 \mathrm{MeV} / \mathrm{m}$, in good agreement.

For the planned RF photo-cathode source upgrade, the proposed beam characteristics change to $\epsilon_{n}=3 \times 10^{-5} \mathrm{~m}$-rad, $N=6 \times 10^{11}$, and $\sigma_{z}=0.75 \mathrm{~mm}$, which gives an estimate of $W_{m}=4 \mathrm{GeV} / \mathrm{m}$. Unfortunately, the formula no longer appiies, as the assumption to this point has been that the plasma response is linear both for the self-focusing and the wake-field amplitude calculations. We have already seen moderate nonlinearities, and while our assumed plasma density has been raised a factor of four, the proposed self-pinched beam is 5000 times denser than before. 
A criterion for determining when one is in the regime where the linear focusing theory will be ifivalid is, with $k_{p} \sigma_{z}=2$, approximately $N r_{e} / \epsilon_{n}>1$, and for the upgraded beam parameters $N r_{e} / \epsilon_{n} \simeq 56$. In this new regime the focusing strength is dependent only on the plasma electron density $n_{c}$ and the equilibrium beam radius is $\sigma_{r_{e} e q}^{2}=\epsilon_{n} / \sqrt{2 \pi r_{e} n_{e} \gamma}=\sqrt{(2 / \gamma)} \epsilon_{n} / k_{p}$. This is an underdense plasma lens regime, ${ }^{[23]}$ and the focusing of the beam should be weaker but much more uniform. If we extrapolate linear wake-field theory to estimate the amplitude of the nonlinear wake-fields we obtain

$$
W_{m} \simeq \frac{N r_{e} m_{e} c^{2}}{2 \sigma_{z}^{2}}\left[-\ln \left(\sqrt{(8 / \gamma)} k_{p} \epsilon_{n}\right)\right]
$$

For the upgraded source parameters we estimate $W_{m}=1.45 \mathrm{GeV} / \mathrm{m}$, with the plasma density $n_{e} \simeq 2.7 \times 10^{14} \mathrm{~cm}^{-3}$, which is within reach of a hollow cathode arc plasma source similar to the one presently used. Note that even though these waves are dominated by radial effects, the maximum wake-field is aimost equal to the so-called wave breaking limit, given by $e E_{w b}=m_{e} c \omega_{p} \simeq \sqrt{n_{e}\left(c: n^{-3}\right)} \mathrm{eV} / \mathrm{m}$ $\simeq 1.65 \mathrm{GeV} / \mathrm{m}$. We thus expect that the wake-fields will display a wealth of interesting nonlinear behavior as well as extremely large accelerating gradients in these experiments.

We gratefully acknowledge the helpful aid or advice given by colleagues at the University of Wisconsin, Fermilab, Los Alamos and UCLA during these investigations. This work supported by the U.S. Dept. of Energy, Division of High Energy Physics, Contract W-31-109-ENG-38. 


\section{REFERENCES}

1. P. Chen, J. M. Dawson, R. W, Huff, and T. Katsouleas, Phys. Rev. Lett., $54,693(1985)$.

2. V. N. Tsytovich, Plasma Astrophysics, Pergamon Press, Oxford (1962).

3. H. Figueroa, W. Gai, R. Konecny, J. Norem, P. Schoessow, and J. Simpson, submitted to Phys. Rev. Lett. (1988).

4. J. B. Rosenzweig, D. B. Cline, B. Cole, H. Figueroa, W. Gai, R. Konecny, J. Norem, P. Schoessow, and J. Simpson, Phys. Rev. Lett. 61, 98 (1988).

5. P. Chen, Particle Accelerators 20 (1985) 171.

6. T. Katsouleas, Phys. Rev. A 33 (1986) 2056.

7. R. D. Ruth, A. Chao, P. L. Morton, and P. B. Wilson, Particle Accelerators 17 (1985) 171.

8. J. B. Rosenzweig, P. Schoessow, B. Cole, W. Gai, R. Konecny, J. Norem and J. Simpson, Phys. Rev. A, (1989).

9. J. B. Rosenzweig, Phys. Rev. Letters, 58 (1987) 555.

10. J. B. Rosenzweig and P. Chen, SLAC-PUB-4571 (1988), to be published in Phys. Rev. D, April 1989.

11. P. Schoessow, submitted to the Proceedings of the 1989 Particle Accelerator Conference, IEEE, Chicago (March 1989).

12. W. Gai, P. Schoessow, B. Cole, R. Konecny, J. Norem, J. B. Rosenzweig and J. Simpson, Phys. Rev. Lett. 61, 2756 (1989).

13. W. K. H. Panofsky and W. A. Wenzel, Rev. Sci. Instr., 27, 967 (1956).

14. R. Keinigs and M. Jones, Physics of Fluids 30, 252 (1987).

15. J. B. Rosenzweig, B. Cole, D. B. Cline, and D. J. Larson, Particle Accelerators, 24, 11 (1988). 
16. D. B. Cline, B. Cole, J. Rosenzweig, and J. Norem, Proceedings of the 1987 Washington Particle Accelerator Conference, IEEE (1987) 154.

17. P. Chen, J.J. Su, T. Katsouleas, J. M. Dawson, R. Keinigs, and M. Jones, IEEE Trans. Plasma Sci.PS-15 (1987).

18. R. Keinigs, M. Jones and J.J. Su IEEE Trans. Plasma Sci., PS-15, 199 (1987).

19. J. B. Rosenzweig, Phys. Rev. A, 38, 3634 (1988).

20. T. Katsouleas and W. B. Mori, Phys. Rev. Lett., 61, 90 (1988).

21. A.Ts. Amatuni, S.S. Sekhpossian and E.V. Elbakian, Yerevan Preprint YerPhi-935(86)-86 Yerevan, USSR (1986).

22. J. B. Rosenzweig, Fermilab Preprint FERMILAR-Pub-88/212 (1989).

23. D. Umstadter, R. Williams, C. Clayton, and C. Joshi,Phys. Rev. Lett., 59 292 (1987).

24. J.J. Su, presented at the Lake Arrowhead Workshop on Advanced Accelerator Concepts.

25. P. Chen, S. Rajagapolan and J. B. Rosenzweig, SLAC-PUB-4746 (October 1988), submitted to Phys. Rev. Lett. 